\title{
Histology of corneal wound healing after deep lamellar endothelial keratoplasty
}

\author{
Chaoran Zhang' \\ Jianjiang $\mathrm{Xu}^{\prime}$ \\ Rongjia Chen ${ }^{2}$ \\ 'Ophthalmology Department, \\ ${ }^{2}$ Pathology Department, Eye and \\ Ear Nose Throat Hospital, Fudan \\ University, Shanghai, China
}

Correspondence: Chaoran Zhang Ophthalmology Department, Eye and Ear Nose Throat Hospital, Fudan University, Shanghai, China

Email zhangchaoran@hotmail.com

\begin{abstract}
Deep lamellar endothelial keratoplasty (DLEK) has become an alternative procedure for bullous keratopathy. Herein, the histopathological characteristics of corneal wound healing after DLEK were reported. A 71-year-old man suffering from left psuedophakia bullous keratopathy received small incision DLEK. Twenty months later, another penetrating keratoplasty (PK) was performed because of the graft failure. The histopathology of the corneal button removed during PK demonstrated the regularity of stromal fiber alignment at the graft interface. Fibrotic repair was limited to the peripheral margins.
\end{abstract}

Keywords: Deep lamellar endothelial keratoplasty, DLEK, bullous keratopathy, penetrating keratoplasty

\section{Introduction}

Over the past several years, deep lamellar endothelial keratoplasty (DLEK) has become an alternative procedure for bullous keratopathy. The published reports on some series have suggested its advantages over standard penetrating keratoplasty (PK) (Terry and Ousley 2005; Huang et al 2007). But the studies about wound healing after DLEK are seldom reported, especially in humans (Melles et al 1998; Lord et al 2006; Kapur et al 2006; Zhang et al 2006).

Here we document the histological findings of a corneal button removed from a patient after DLEK.

\section{Case report}

A 71-year-old man suffering from left psuedophakia bullous keratopathy received a small incision ( $5 \mathrm{~mm}$ length) DLEK with $7.75 \mathrm{~mm}$ recipient bed. The surgical procedures were as previously described (Terry and Ousley 2000). Because the patient had angle closure glaucoma with controlled intraocular pressure (IOP) after trabeculectomy, it was a little difficult to open the folded donor disc in the shallow anterior chamber. The first day after DLEK, the donor disc dislocated. An air bubble was injected into the anterior chamber to reposition and support the donor disc in the recipient bed. However, the disc was seen to overlie the inferior edge of the recipient bed. Despite this, the donor disc kept adherence to and cleared the recipient's cornea.

Six months after DLEK, the patient's best corrected visual acuity (BSCVA) was $20 / 80$ with a manifested refraction of $-1.00+1.50 \times 80$. Endothelial cell count was 1309 cells $/ \mathrm{mm}^{2}$. The cornea was transparent with some haze at the graft interface.

Sixteen months after DLEK, the patient's BSCVA was decreased to 20/200. Cornea edema was observed under slit-lamp biomicroscope. There was no significant inflammation such as keratic precipitates (KP) on the endothelium or increased cells in the anterior chamber. The endothelial cell count was 905 cells $/ \mathrm{mm}^{2}$. Graft failure was considered. 
Twenty months after DLEK, the patient's BSCVA was finger-counting. Corneal edema was more severe. Therefore, $7.75 \mathrm{~mm}$ PK was performed.

The removed corneal button was immersed in $10 \%$ neutral buffered formalin and sent for histological evaluation. The corneal tissue was stained with hematoxylin-eosin and periodic acid-Schiff, but no special findings were obtained with PAS stain (data not shown). The epithelium of the button was almost normal with the exception of some focal bullous separation from the Bowman layer. The stromal lamellae were parallel. More thickened and compacted collagen fibers presented in the posterior stroma. The host - graft interface was identified under light microscope (Figures 1, 2). They both attached, other than one peripheral area, where the anterior and posterior stroma dehisced. At the same specimen, the posterior lamella was longer than the anterior one. Between them, a piece of Descemet's membrane and stromal fragment manifesting as an amorphous structure was presented (Figure 3). The keratocytes were located between the lamellae. They had thin nuclei, ill-defined borders. Increased cells were found at one posterior edge (Figure 4). The Descemet's membrane exhibited regularly and continuously without any folds or tears. The endothelium cell layer was attenuated (Figure 1).

\section{Discussion}

Because human corneas can only be seldom analyzed following DLEK, histological knowledge about corneal wound healing related to this surgical procedures is limited. As previously reported, the fibrosis was mainly noted around the region connecting donor and recipient edges. At the interface plane, no demonstrable fibrosis was found on light microscope (Lord et al 2006). Sometimes, electron microscope revealed changes of keratocytes at the interface, consequent on engulfing red blood cells or iris pigments left during the surgery (Lord et al 2006). In our case, the histological characteristics were partially similar to other authors' reports.

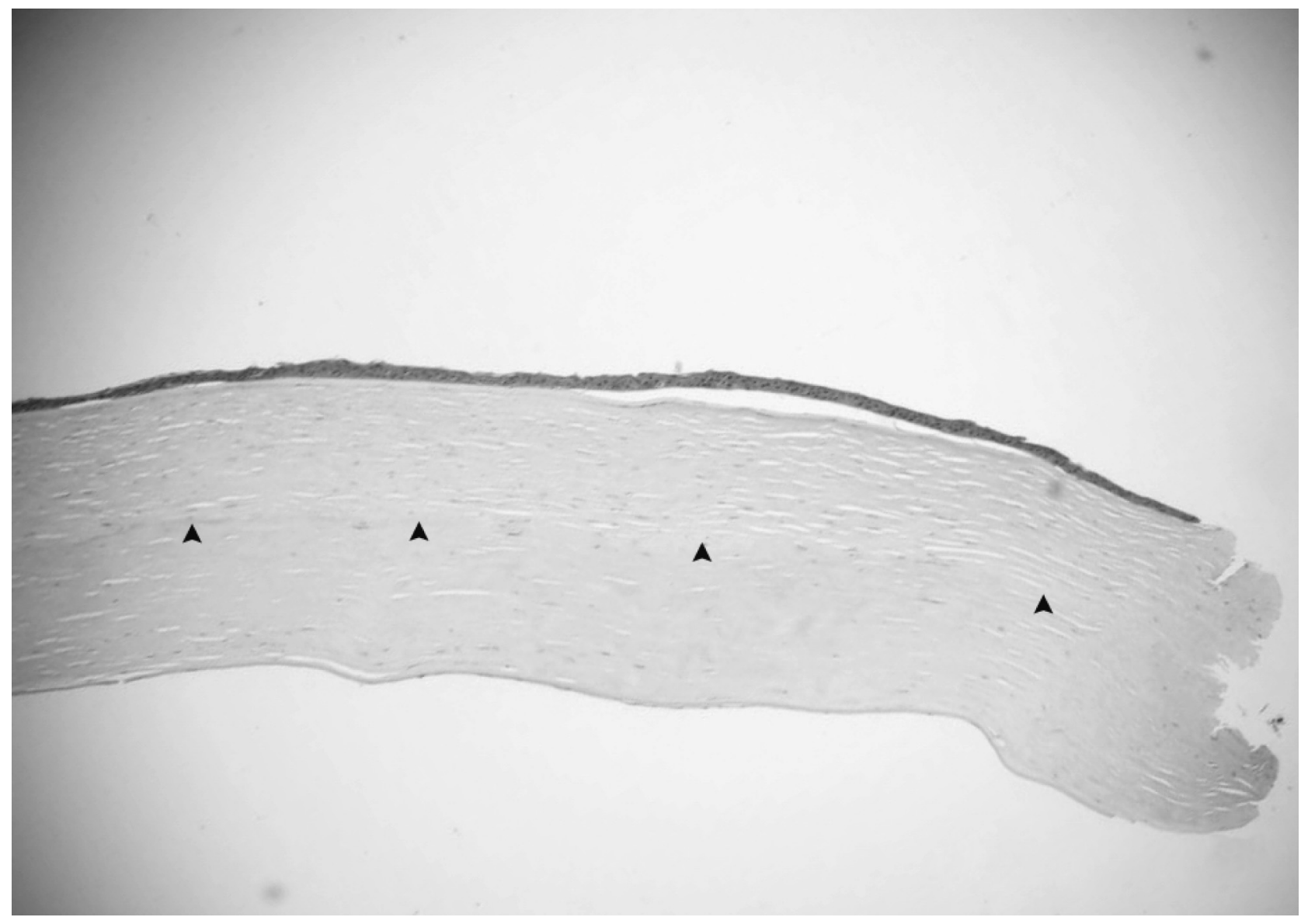

Figure I Light photomicrographs of histological section obtained from the periphery of the corneal button (hematoxylin and eosin stain). Photomicrograph showing detached epithelial layer and attenuated endothelial layer.Arrows indicate the interfaced between the host and donor stroma. Original magnification $\times 40$. 


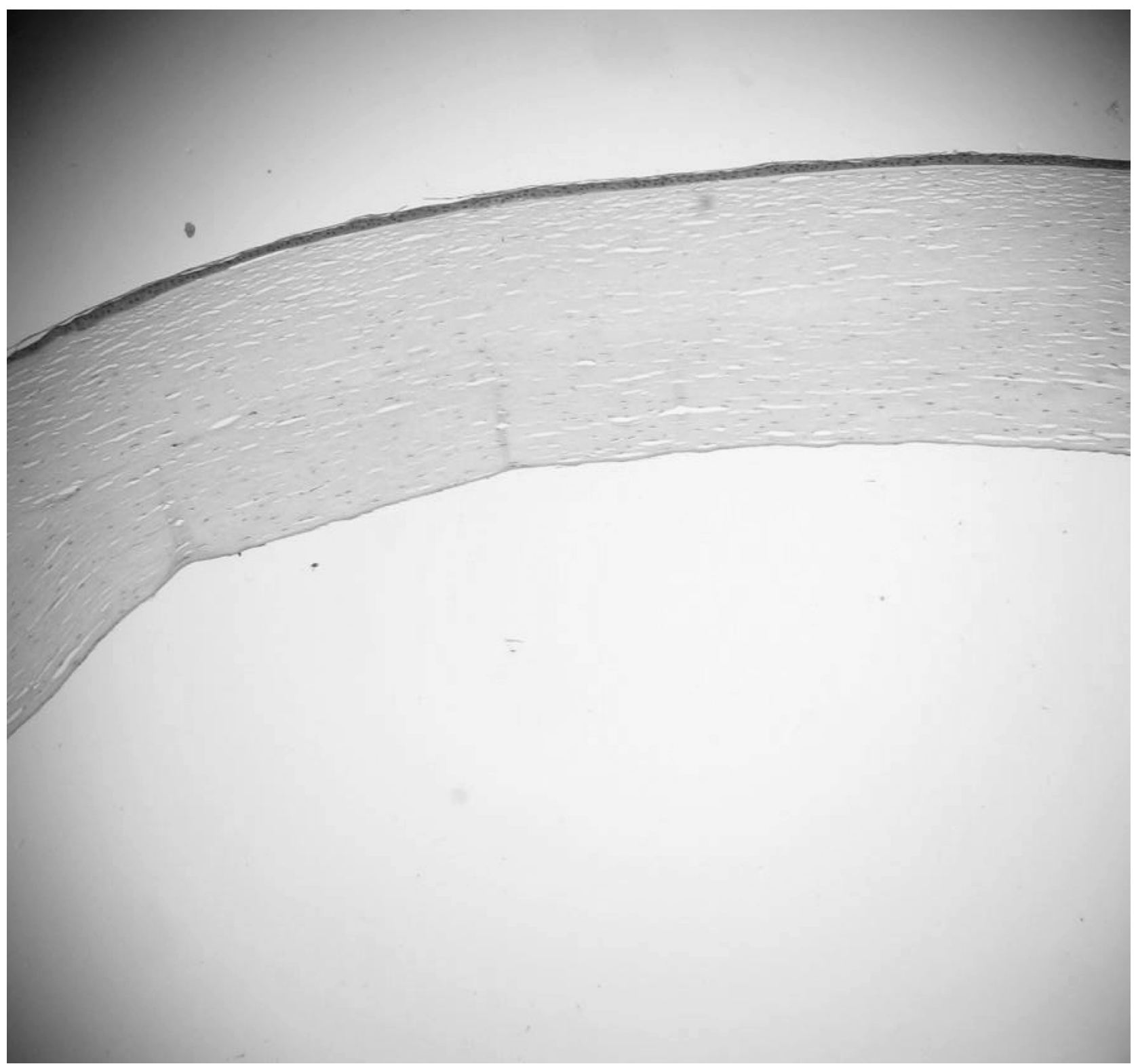

Figure 2 Light photomicrographs of histological section obtained from the center of the corneal button (hematoxylin and eosin stain). Photomicrograph showing attached corneal stoma. Original magnification $\times 40$.

Generally speaking, there were two different injuries to both host and donor corneas involved in DLEK (Kapur et al 2006; Lord et al 2006; Zhang et al 2006). At the graft interface, the injury was lamellae separation parallel to the corneal surface, almost without collagen fiber rupture. But at the peripheral circumference, the injury was vertical to the corneal surface, which broke posterior stromal and Descemet's membrane. In harmony with these injuries, corneal wound healing procedures underwent different ways. At the peripheral area, because of the collagen fibers rupture, the stromal margins healed in a manner similar to that occurring after standard PK. Briefly, fibroblasts from corneal stroma formed a tenuous scar along the wound lips to seal the gap between the donor and host troma. The newly formed scar had a tendency to remodel over time. In our study, because the recipient bed of PK was as the same size as that of DLEK, we could not get an overview of wound healing involving peripheral circumference. The increased cells at one posterior edge traced the scar formed in the earlier stage post operation. At the graft interface, the wound healing procedure was in the other way. No newly formed fibers inserting vertically into the interface could be observed under light microscopy. The lamellae aligned regularly, as shown in our study. The interface plane could still be identified. That meant there 


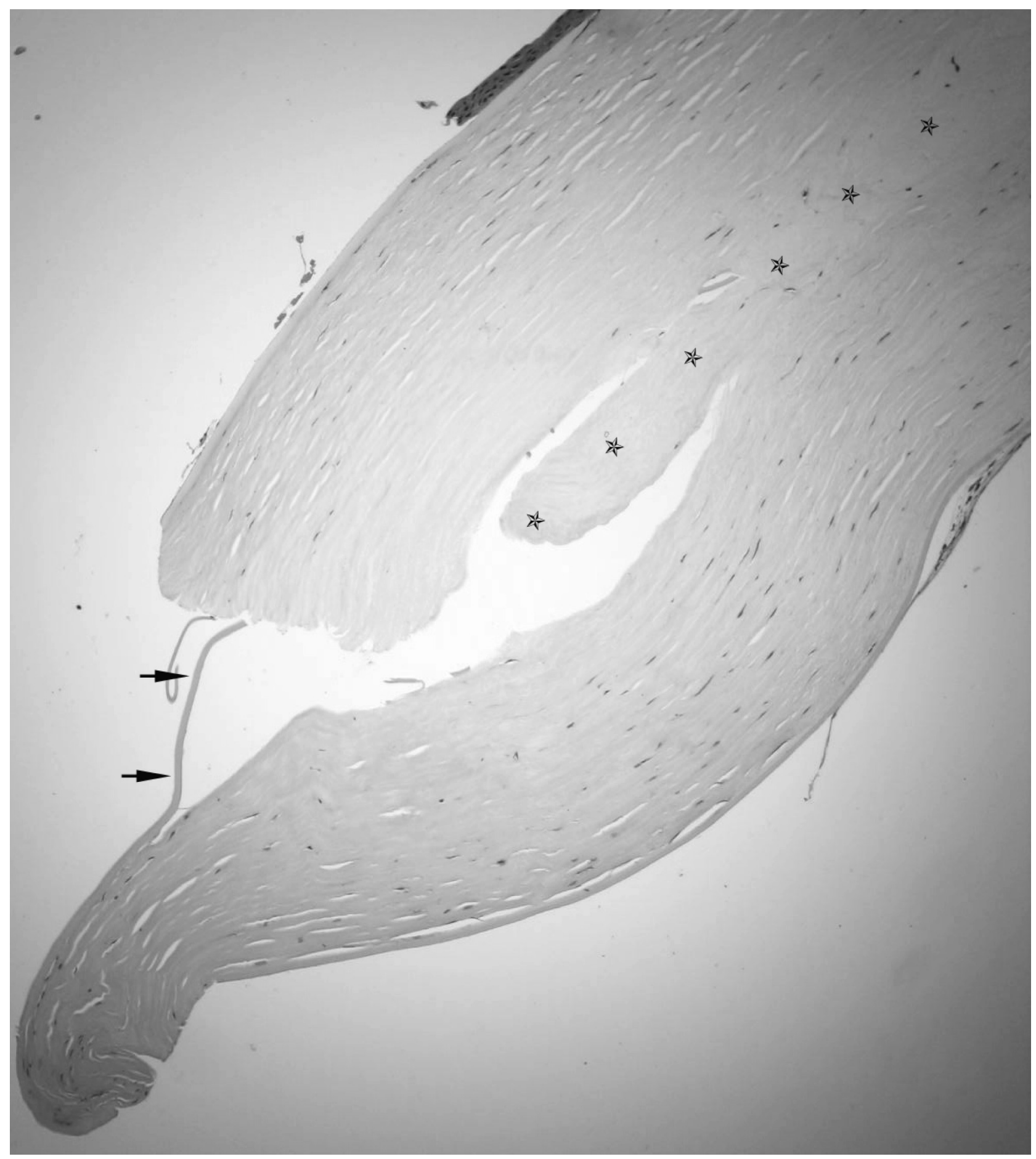

Figure 3 Light photomicrographs of histological section obtained from the periphery of the corneal button (hematoxylin and eosin stain). Photomicrograph showing stroma separation with a piece of Descemet's membrane (arrows) and an amorphous fragment (stars). Original magnification $\times 100$.

was some haze at the interface, which was caused by manual cutting during recipient bed preparation. Overtime, stromal remodeling may eventually make the interface unremarkable. The perfect smooth interface can only be obtained with femtosecond laser technique. To some extent, the corneal wound healing of DLEK was similar to that of laser in situ keratomileusis (LASIK) flap (Ivarsen et al 2003). Dehiscence of the flap many years after LASIK indicates that it would not be too difficult to remove the donor disc from the recipient bed years after DLEK (Iskander et al 2001). Thus another DLEK can be chosen following a failed one (Terry and Ousley 2006). 


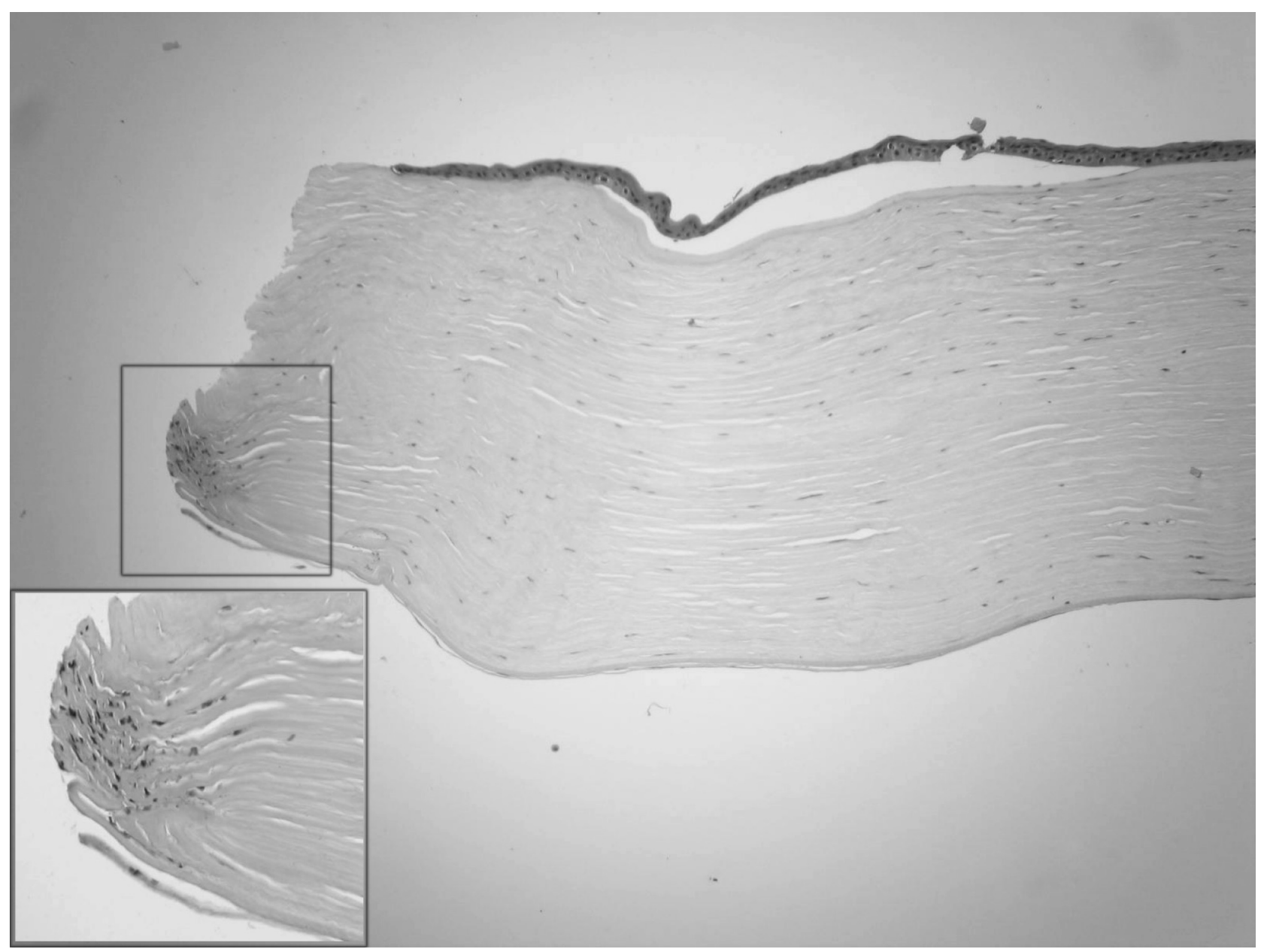

Figure 4 Light photomicrographs of histological section obtained from the periphery of the corneal button (hematoxylin and eosin stain). Photomicrograph showing increased keratocytes at the posterior edge. Original magnification $\times 100$. Inserted figure manifested high magnification $\times 200$.

In our case, a tongue-shaped stroma was inserted between the host and donor lamellae. In our experience, because it was more difficult for surgeon to cut the inferiorly posterior stroma, the residual fragments were usually seen at 6:00 o'clock in marginal recipient bed. During the wound remodeling procedure, the fragment may gradually change to amorphous structure. At the same place, the donor disc extended beyond and overlaid the recipient bed edge. Thereafter, a piece of Descemet's membrane was pulled down during PK.

In conclusion, the histopathology of the corneal button demonstrated the regularity of stromal fiber alignment at the graft interface. Fibrotic repair was limited to the periphery margins. The irregular marginal structure was the result of both collagen fiber repair and inappropriate surgical manipulation.

\section{References}

Huang T, Chen JQ, Wang YJ, et al. 2007. Preliminary clinical study of sutureless, small-incision deep lamellar endothelial keratoplasty surgery. Zhonghua Yan Ke Za Zhi, 43:118-23.
Iskander NG, Peters NT, Anderson Penno E, et al. 2001. Late traumatic flap dislocation after laser in situ keratomileusis. $J$ Cataract Refract Surg, 27:1111-14.

Ivarsen A, Laurberg T, Møller-Pedersen T. 2003. Characterisation of corneal fibrotic wound repair at the LASIK flap margin. Br J Ophthalmol, 87:1272-8.

Kapur R, Sugar J, Edward DP. 2006. Deep lamellar endothelial keratoplasty: histopathology of complications in initial cases. Cornea, 25:851-4.

Lord RK, Price FW, Price MO, et al. 2006. Histology of posterior lamellar keratoplasty. Cornea, 25:1093-6.

Melles GR, Eggink FA, Lander F, et al. 1998. A surgical technique for posterior lamellar keratoplasty. Cornea, 17:618-26.

Terry MA, Ousley PJ. 2000. Small-incision deep lamellar endothelial keratoplasty (DLEK): six-month results in the first prospective clinical study. Cornea, 24:59-65.

Terry MA, Ousley PJ. 2005. Deep lamellar endothelial keratoplasty visual acuity, astigmatism, and endothelial survival in a large prospective series. Ophthalmology, 112:1541-8.

Terry MA, Ousley PJ. 2006. Deep lamellar endothelial keratoplasty: early complications and their management. Cornea, 25:37-43.

Zhang CR, Xu JJ, Chen RJ, et al. 2006. Histopathological study of corneal wound healing in guinea pigs after deep lamellar endothelial keratoplasty with small incision. Zhonghua Yan Ke Za Zhi, 42:694-8. 
